\title{
Application of higher-order spectral analysis to local field potentials recorded in patients treated with deep brain stimulation
}

\author{
Sara Marceglia, Anna Maria Bianchi, Member, IEEE, Guglielmo Foffani, Alberto Priori, \\ Sergio Cerutti, Fellow, IEEE
}

\begin{abstract}
Local field potentials (LFPs) recorded from implanted deep brain electrodes demonstrated the oscillatory nature of human basal ganglia. LFP rhythms were mainly characterized by means od power spectral analysis, thus loosing information related to rhythm phase synchronization and to event related phase modulations. Because the application of higher-order spectral analysis methodology can overcome such limitation, here we review the present applications of bispectral and cross-bispectral analysis to LFP recordings. The results obtained up to now showed that higher-order spectral analysis was able to clarify detect different rhythm synchronizations and interactions characterizing different pathologies and patient's states.
\end{abstract}

\section{INTRODUCTION}

The availability of local field potential (LFP) recordings from patients with implanted deep brain stimulation (DBS) electrodes opened the way to the investigation of oscillationbased alternatives to the classical basal ganglia model[1], [2]. LFP analysis was used to assess the functional properties of these deep neural structures in response to various pharmacological and behavioral stimuli. LFPs have revealed the oscillatory behavior of deep brain structures and can detect focal network rhythms that are not necessarily observable in single neurones or neurone pairs [1], [3]. Two or three days after surgery for electrodes positioning and before the connection of electrode wires to the subcutaneous electrical stimulator, DBS electrodes are accessible for recording LFPs that can be defined as the synchronous presynaptic and postsynaptic activity of large neuronal populations. Oscillations in the basal ganglia were mainly studied in Parkinson's disease (PD) and were disclosed to range in a wide interval, from low frequencies (below $7 \mathrm{~Hz}$ ), to frequencies in the beta band $(13-35 \mathrm{~Hz})$, up to high frequencies, around $70 \mathrm{~Hz}$ and around $300 \mathrm{~Hz}$ [3], [4]. LFP rhythms are modulated by dopamine intake, by voluntary movement execution and imagination, are related to the

S. Marceglia is with the Dipartimento di Ingegneria e Architettura, Università degli Studi di Trieste, Trieste, Italy (e-mail: smarceglia@units.it).

AM Bianchi* and S Cerutti are with the Dipartimento di Elettronica, Informazione e Bioingegneria, Politecnico di Milano, Milano, Italy (e-mail: annamaria.bianchi@polimi.it; sergio.cerutti@polimi.it; *corresponding author: tel +39 022399 3342; fax: +39 022399 3360).

A. Priori is with the Clinical Center for Neurostimulation, Neurotechnology, and Movement Disorders, Fondazione IRCCS Ca'Granda Ospedale Maggiore Policlinico and Università degli Studi di Milano, Milano, Italy (e-mail: alberto.priori@unimi.it).

G. Foffani is with the Hospital Nacional de Parapléjicos, SESCAM, Toledo, Spain (e-mail: gf32@drexel.edu) patient's clinical and motor state, and different pathologies are characterized by different LFP patterns [5], [6].

However, traditional methodological approaches of signal processing cannot detect information related to rhythm phase synchronization and to event related phase modulations. The application of different methodological approaches, such as higher-order analysis, could help clarifying the role played by rhythms in the information processing when complex mechanisms, such as cross-frequency coupling, are present [7].

Most studies defined the LFP rhythms in the human basal ganglia by means of power spectral analysis. The power spectrum, that is related directly to the second order statistic, can completely describe the signal when the underlying process is Gaussian and linear. When non-linear correlations between rhythms are present, whereas power-spectral analysis suppresses phase relations between oscillations, the third order spectrum (bispectrum) does contain such information and it is particularly useful in the detection of phase-locking phenomena[8], [9]. When the processes characterizing the system are more than one, the crossbispectrum can detect phase locking phenomena between different signals. In biomedicine, the bispectrum was applied, for the first time, to bioignals for investigating EEG recordings [10]. From that first work, the bispectrum and cross-bispectrum were used to charachterize biological signals and it has been recently proposed for emotional state classification [11], the identification of Alzheimer's disease EEG biomarkers[12], the study of short-memory processing [13], the detection of cardiac ischemia[14], and many others.

The application of these methodological approaches to LFPs could open the possibility to uncover non-linear dynamics of brain cortical and subcortical oscillations. In this paper, we review the present applications of bispectral and cross-bispectral analysis to LFP recordings and propose a possible pathophysiological interpretation model of the results obtained.

\section{BISPECTRAL ANALYSIS AND NON-LINEAR LFP DYNAMICS}

\section{A. LFP bispectral biomarkers in Parkinson's Disease}

The DBS target for PD patients is the subthalamic nucleus (STN). LFP recordings from the STN of PD patients were extensively described in different clinical states and are now used as control variable in new adaptive DBS strategies[6].

Higher-order spectral analysis (bispectrum and bicoherence) was applied to investigate non-linear 
correlations between STN-LFP rhythms in PD before and after the administration of a clinical effective dose of levodopa[15]. The bispectrum is used to detect phase synchronizations among oscillations in a single signal. A bispectral peak at frequencies (f1, f2) implies that the two oscillations, one at $\mathrm{fl}$ and one at $\mathrm{f} 2$, interact and that the oscillation at $\mathrm{f} 1+\mathrm{f} 2$ in the signal is the result of this non-linear interaction. The bispectrum is defined as the two-dimensional Fourier Transform (FT) of the third-order cumulant.

The results disclosed that Parkinson's disease produces non-linear correlations between STN LFP rhythms oscillating at different frequencies[15], differing between genders [16], and characterizing levodopa-induced dyskinesias [17].

The most marked non-linear correlation phenomenon was detected between the low-beta $(13-20 \mathrm{~Hz})$ and the high beta (20-35 Hz) rhythm: the LFP activity in the high-beta range (20-35 Hz) is distorted by a harmonic of the low-beta rhythm. When patients were in the off state, non-linear correlations were also observed between the low-beta rhythm and other rhythms at very-low frequencies $(2-7 \mathrm{~Hz})$ or in the alpha range $(8-12 \mathrm{~Hz})$. After levodopa administration, these nonlinear correlations decrease and the low-beta/high-beta distortion is virtually eliminated: levodopa increased nonlinear interactions within the low-frequency band and decreased (or left unchanged) all the other non-linear interactions, thereby increasing segregation between LFP rhythms operating at different frequencies. Hence, after the administration of a clinically effective levodopa dose, the system moves towards a more segregated state[15].

Bispectral analysis in PD was also applied to investigate gender-related differences in the non-linear phase synchronizations between LFP rhythms. LFPs were in fact shown to be gender-specific, with women displaying higher beta activity than men before levodopa administration. The pathophysiological basis of gender differences in PD may reside in the different effects of gonadal hormones on the brain development and functioning (Marceglia et al 2007). The results showed that, in the absence of levodopa stimulation, males had higher synchronization within the low-frequency band, whereas the pattern of synchronizations between and within the other rhythms was similar. After dopaminergic therapy had restored more physiological dopamine levels, the non-linear phase coupling phenomena were similar between the two genders[16].

In $\mathrm{PD}$, after the administration of levodopa, there is a 30 minutes time window after which patients first improve in rigidity thanks to the effect of levodopa (non-dyskinetic on) and, then, can show involuntary movements due to peak-dose levodopa (dyskinetic on). The oscillatory activity during levodopa-induced dyskinesias is characterized by a large increase in the low-frequency $(2-7 \mathrm{~Hz})$ band (Alonso-Fretch et al, 2006). Bispectral analysis showed that, after the administration of levodopa, without diskinesias, the lowfrequency oscillation increased, whereas the bispectral power in the region $(2-7 \mathrm{~Hz}, 2-7 \mathrm{~Hz})$ did not change, suggesting that the low-frequency oscillation was boosted by levodopa administration without changing its non-linear properties. Conversely, the low-beta oscillation decreased together with the decrease in the bispectral power in the region $(13-20 \mathrm{~Hz}$, $13-20 \mathrm{~Hz}$ ), but did not completely disappear. When the

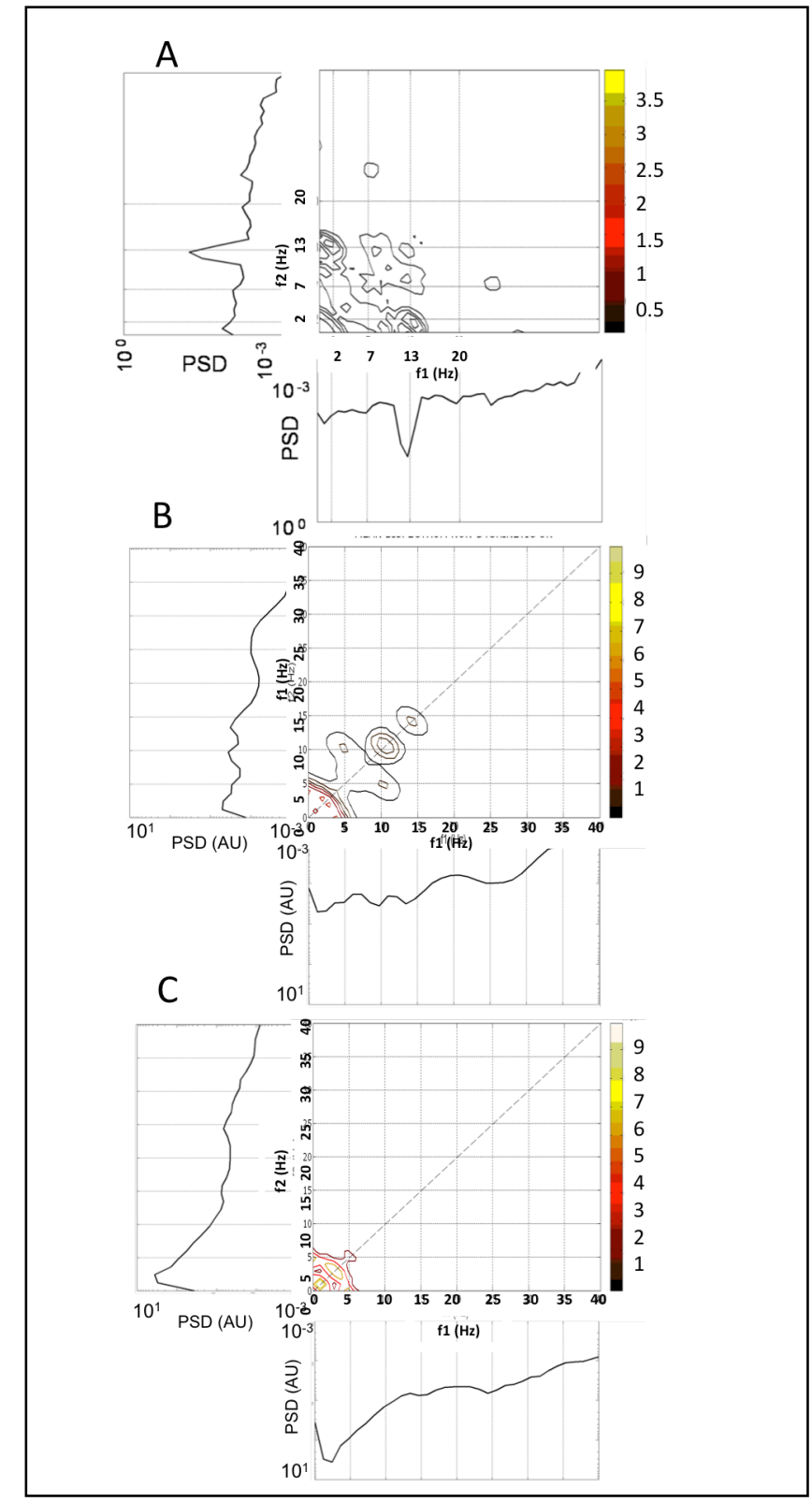

Figure 1. Example of bispectrum estimated in STN-LFPs before levodopa administration (A), and after levodopa administration without (B) and with (C) levodopa-induced dyskinesias. On the $\mathrm{x}$ and $\mathrm{y}$ axis are represented power spectra (log PSD).

patients displayed levodopa-induced dyskinesias, there was an abnormal increase of low-frequency oscillations, together with an increase in bispectral power in the region (2$7 \mathrm{~Hz}, 2-7 \mathrm{~Hz}$ ). In addition, the low-beta oscillation decreased more and the rhythms became totally segregated and all the peaks in the bispectral region $(13-20 \mathrm{~Hz}, 13-20 \mathrm{~Hz})$ disappeared (Figure 1) [17].

\section{B. LFP bispectral biomarkers in Dystonia}

Dystonic patients are implanted with DBS electrodes in the Globus Pallidus internus (GPi). Spectral analysis of GPi LFPs from dystonic patients at rest revealed power spectra dominated by the oscillatory activity below $7 \mathrm{~Hz}$, in the lowfrequency band, whereas activity in the beta band is almost inexistent. Bispectral and bicoherence analysis applied to GPi 
LFPs recorded in dystonic patients [18] disclosed that the low-frequency band generates a second harmonic, without any other non-linear interaction between rhythms. This result suggested that the rhythms expressed in the GPi of the untreated PD patient are similar to those observed in the STN and are characterized by a large amount of nonlinear interactions. Conversely, in the dystonic GPi, as well as in the parkinsonian GPi after dopaminergic medication, the rhythms are substantially independent.

\section{APPLICATIONS OF CROSS-BISPECTRAL ANALYSIS}

Cross-bispectral analysis was applied to LFP and electromyographic (EMG) signals recorded in a patient with essential tremor, implanted in the subthalamic nucleus (STN) with DBS electrodes[19]. Whereas the bispectrum is able to characterize non-linear interactions and phase synchronizations between the rhythms composing a single signal, the cross-bispectrum $\left(\mathrm{C}_{\mathrm{xyz}}\right)$ is able to detect such interactions between two or three signals. As well as the bispectrum, the cross-bispectrum is defined as the twodimensional Fourier Transform (FT) of the third-order crosscumulant.

LFPs were recorded at rest synchronously with the surface electromyography (EMG) of the limbs showing tremor two days after functional neurosurgery for DBS electrode placement.

The power spectrum showed that both the LFP and the EMG had a predominant peak at $5 \mathrm{~Hz}$ and a smaller peak at $10 \mathrm{~Hz}$, and had a high cross-coherence at $5 \mathrm{~Hz}$ and $10 \mathrm{~Hz}$. The LFP signal also showed the characteristic STN high-beta activity (25-35 Hz). Bispectral analysis was applied to STN.

LFPs and showed that STN rhythms were independent. Because in physiological application there is no a priori knowledge on the kind of relationship between signals, simulations were needed to uncover the specific pattern of non-linear synchronizations from the distribution of crossbispectral peaks in the bidimensional frequency domain (Marceglia NER 09). Results from simulations were used, to infer, from the position of symmetric peaks in the principal domain, the reciprocal relationship between the analyzed signals.

The cross-bispectrum disclosed significant non-linear synchronizations between LFPs and EMG. Since the crossbispectrum was calculated among two signals, the $\mathrm{C}_{\mathrm{xyy}}$ configuration for cross-cumulant estimation was chosen,

Figure 2. Cross-bispectral analysis in essential tremor. where $\mathrm{x}$ corresponded to the LFP and y corresponded to the EMG. The cross-bicoherence (normalized crossbispectrum) displayed two peaks in the principal domain at $(5 \mathrm{~Hz}, 5 \mathrm{~Hz})$ and $(10 \mathrm{~Hz},-5 \mathrm{~Hz})$. This pattern of interaction was similar to that observed in a simulation study (Marceglia NER09), and suggested that the $5 \mathrm{~Hz}$ subthalamic rhythm and the $5 \mathrm{~Hz}$ muscular rhythm were non-linearly interacting and that this interaction produced at least part of the $10 \mathrm{~Hz}$ oscillation in the EMG. The $10 \mathrm{~Hz}$ oscillation in the LFP was independent and not involved in the resonance system probably causing the cortico-muscular non linear coherence.

\section{A POSSIBLE MODEL OF BASAL GANGLIA MOTOR OSCILLATIONS}

According to these observations from real recordings, a possible model of basal ganglia motor oscillations in Parkinson's disease without dopaminergic stimulation, Parkinson's disease with dopaminergic stimulation without dyskinesias, and in hyperkinesias (Dystonia, dyskinesias, tremor) is proposed in Figure 3.

The dopamine-dependent non-linear interaction between the low-beta and the high-beta rhythms could reflect the phaselocked activity of two generators. In BG, the STN and the globus pallidus externus (GPe) form a closed-loop that can sustain oscillations in the beta range [20] as well as in the low-frequency range. A possible explanation for the observed bispectral pattern is that the phase relations between rhythms in the STN could be the result of a nonlinear synchronization between STN and GPe that induces the phase coupling in the low-beta range, thereby affecting the BG output (Figure 3A). This phenomenon is abolished by the administration of levodopa that restores the normal independence between generators and normalizes, at least in the beta range, the BG output. Also, the abnormal low-beta activity is drastically reduced but not completely destroyed; however, the reduction allows the non-spreading of the lowbeta throughout the circuit; on the other hand, the lowfrequency rhythm is restored and its non-linear interaction at the STN and GPi level appears, too (Figure 3B). Finally, if the dopaminergic or electrical stimulation leads to dyskinesias in Parkinson's disease, or if the patient is affected by dystonia, the low-frequency rhythm is abnormally increased and non-linear synchronizations spread from STN to GPi, leading to hyperkinesias (Figure 3C).

If there is tremor, a pathological synchronization at the tremor frequency involves the STN and the muscles where a
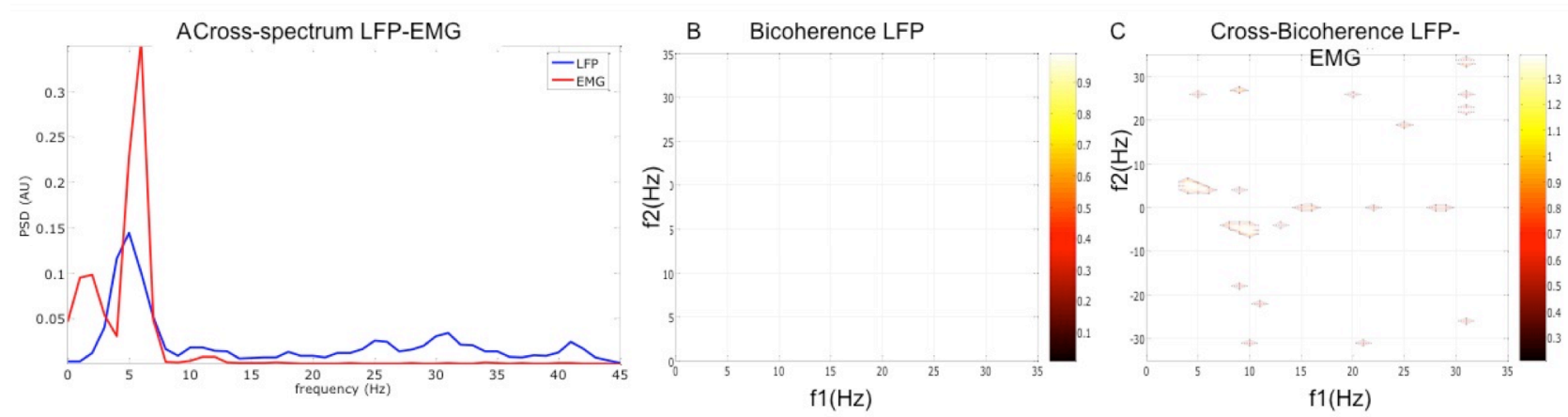


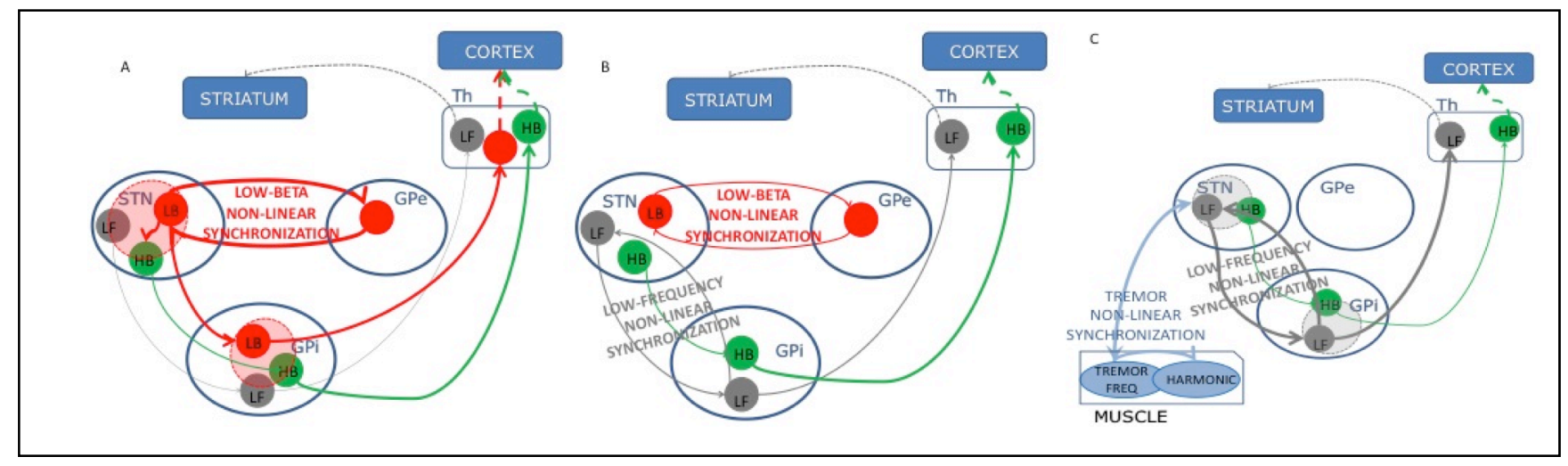

Figure 3. Possible model of basal ganglia rhythm interactions (A) in PD before levodopa; (B) in PD after levodopa; (C) in hyperkinesias. Th = Thalamus; $\mathrm{STN}=$ Subthalamic Nucleus; $\mathrm{GPi}=$ Globus Pallidus internus; $\mathrm{GPe}=$ Globus Pallidus externus; $\mathrm{LF}=\mathrm{LFP}$ low-frequency rhythms; $\mathrm{LB}=$ LFP low-beta rhythm; HB = LFP high-beta rhythm

double harmonic is also produced. In all cases, the CM-PfVO thalamic complex expresses a low-frequency rhythm, possibly reverberating to the striatum.

\section{CONCLUSIONS}

From a signal processing point of view, the investigation of nonlinear interactions can help to correctly interpret the power spectrum. The knowledge gained with the bispectrum permits, if not a real quantification, at least a qualitative distinction between the independent activity of LFP rhythms and the synchronized activity due to the interaction between rhythms. From a pathophysiological point of view, despite the suggested role of interactions between rhythms, their origin remains debatable. Non-linear interactions could reflect the synchronization between two or more generators. LFPs generators are not single neurons, but populations of synchronized neurons. Therefore, the interaction between rhythms could be due to a common network input, able to synchronize the output of the neural networks generating LFPs. However, it cannot be a priori excluded the possibility that the observed non-linearity could be the result of a nonlinear behavior of a single oscillator.

In conclusion, higher-order spectral analysis clarified that in different pathologies and patient's states, LFP rhythms behave differently and display different synchronizations, and interactions.

\section{REFERENCES}

[1]P. Brown and D. Williams, "Basal ganglia local field potential activity: character and functional significance in the human," Clin. Neurophysiol. Off. J. Int. Fed. Clin. Neurophysiol., vol. 116, no. 11, pp. 2510-2519, 2005. [2] S. Marceglia, L. Rossi, G. Foffani, A. Bianchi, S. Cerutti, and A. Priori, "Basal ganglia local field potentials: applications in the development of new deep brain stimulation devices for movement disorders," Expert Rev. Med. Devices, vol. 4, no. 5, pp. 605-614, Sep. 2007.

[3]A. Priori, G. Foffani, A. Pesenti, F. Tamma, A. M. Bianchi, M. Pellegrini, M. Locatelli, K. A. Moxon, and R. M. Villani, "Rhythm-specific pharmacological modulation of subthalamic activity in Parkinson's disease," Exp. Neurol., vol. 189, no. 2, pp. 369-379, Oct. 2004.

[4]G. Foffani, A. Priori, M. Egidi, P. Rampini, F. Tamma, E. Caputo, K. A. Moxon, S. Cerutti, and S. Barbieri, " $300-\mathrm{Hz}$ subthalamic oscillations in Parkinson's disease," Brain J. Neurol., vol. 126, no. Pt 10, pp. 2153-2163, Oct. 2003.

[5]S. Marceglia, M. Fumagalli, and A. Priori, "What neurophysiological recordings tell us about cognitive and behavioral functions of the human subthalamic nucleus," Expert Rev. Neurother., vol. 11, no. 1, pp. 139-149, Jan. 2011.

[6]A. Priori, G. Foffani, L. Rossi, and S. Marceglia, "Adaptive deep brain stimulation (aDBS) controlled by local field potential oscillations," Exp. Neurol., vol. 245, pp. 77-86, Jul. 2013.

[7] V. Jirsa and V. Müller, "Cross-frequency coupling in real and virtual brain networks," Front. Comput. Neurosci., vol. 7, p. 78, 2013.

[8]C. L. Nikias and J. M. Mendel, "Signal processing with higher-order spectra," IEEE Signal Process. Mag., vol. 10, no. 3, pp. 10-37, Jul. 1993. [9]D. Brillinger and M. Rosenblatt, "Computation and interpretation of kth order spectra," in Spectral Analysis of Time Series, John Wiley \& Sons, 1967.

[10] G. Dumermuth, P. J. Huber, B. Kleiner, and T. Gasser, "Analysis of the interrelations between frequency bands of the EEG by means of the bispectrum. A preliminary study," Electroencephalogr. Clin. Neurophysiol., vol. 31, no. 2, pp. 137-148, Aug. 1971

[11] R. Yuvaraj, M. Murugappan, N. M. Ibrahim, K. Sundaraj, M. I. Omar, K. Mohamad, and R. Palaniappan, "Optimal set of EEG features for emotional state classification and trajectory visualization in Parkinson's disease," Int. J. Psychophysiol. Off. J. Int. Organ. Psychophysiol., vol. 94, no. 3, pp. 482-495, Dec. 2014.

[12] R. Wang, J. Wang, S. Li, H. Yu, B. Deng, and X. Wei, "Multiple feature extraction and classification of electroencephalograph signal for Alzheimers' with spectrum and bispectrum," Chaos Woodbury N, vol. 25, no. 1, p. 013110 , Jan. 2015

[13] B. Schack, N. Vath, H. Petsche, H.-G. Geissler, and E. Möller, "Phase-coupling of theta-gamma EEG rhythms during short-term memory processing," Int. J. Psychophysiol. Off. J. Int. Organ. Psychophysiol., vol. 44, no. 2, pp. 143-163, May 2002.

[14] A. Al-Fahoum, A. Al-Fraihat, and A. Al-Araida, "Detection of cardiac ischaemia using bispectral analysis approach," J. Med. Eng. Technol., vol. 38, no. 6, pp. 311-316, Aug. 2014.

[15] S. Marceglia, G. Foffani, A. M. Bianchi, G. Baselli, F. Tamma, M. Egidi, and A. Priori, "Dopamine-dependent non-linear correlation between subthalamic rhythms in Parkinson's disease," J. Physiol., vol. 571, no. Pt 3, pp. 579-591, Mar. 2006.

[16] S. Marceglia, A. M. Bianchi, S. Mrakic-Sposta, G. Foffani, A. Priori, and S. Cerutti, "Gender-related differences in non-linear phase synchronizations between subthalamic rhythms in Parkinson's disease," 2007, pp. 522-525.

[17] S. Marceglia, A. Leone, G. Foffani, F. Cogiamanian, S. MrakicSposta, F. Tamma, and A. Priori, "Neurophysiological correlates of parkinsonian dyskinesias in subthalamic oscillatory activity," Mov. Disord. Off. J. Mov. Disord. Soc., vol. 22, p. S103, 2007.

[18] S. Marceglia, A. M. Bianchi, G. Baselli, G. Foffani, F. Cogiamanian, N. Modugno, S. Mrakic-Sposta, A. Priori, and S. Cerutti, "Interaction between rhythms in the human basal ganglia: application of bispectral analysis to local field potentials.," IEEE Trans. Neural Syst. Rehabil. Eng. Publ. IEEE Eng. Med. Biol. Soc., vol. 15, no. 4, pp. 483-492, Dec. 2007.

[19] S. Marceglia, A. M. Bianchi, S. Cerutti, and D. Servello, "Crossbispectral analysis of local field potentials: An application to essential tremor," 2009, pp. 494-497.

[20] M. D. Bevan, P. J. Magill, D. Terman, J. P. Bolam, and C. J. Wilson, "Move to the rhythm: oscillations in the subthalamic nucleus-external globus pallidus network," Trends Neurosci., vol. 25, no. 10, pp. 525-531, Oct. 2002. 